Notfall Rettungsmed 2017 $\cdot 20: 469$

DOI 10.1007/s10049-017-0345-z

Online publiziert: 17. August 2017

(c) Springer Medizin Verlag GmbH 2017

CrossMark

\section{Waydhas' ${ }^{1}$ B. W. Böttiger ${ }^{2} \cdot$ C. Wrede ${ }^{3}$}

'Klinik und Poliklinik für Unfallchirurgie, Universitätsklinikum Essen, Essen, Deutschland ${ }^{2}$ Klinik für Anästhesiologie und Operative Intensivmedizin, Universitätsklinikum Köln (AöR), Köln, Deutschland

${ }^{3}$ Notfallzentrum mit Rettungsstelle, HELIOS Klinikum Berlin-Buch, Berlin, Deutschland

\title{
Hoher Impact - am besten im Original
}

Warum soll ich Originalarbeiten in der Notfall+Rettungsmedizin lesen? Warum soll ich sie dort publizieren?

Schließlich ist die Notfall+Rettungsmedizin ein Weiterbildungsforum, zeigt Übersichten und aktuelle Entwicklung im Rahmen der Themenhefte und deckt alle Bereiche der präklinischen, innerklinischen, ärztlichen und nichtärztlichen, individualmedizinischen und organisatorischen Notfallmedizin ab.

Unsere Zeitschrift ist dabei auch ein Ort, an dem sich zahlreiche rettungsdienstliche Organisationen präsentieren und an dem hochaktuelle neue Entwicklungen, Konzepte und Herausforderungen vorgestellt und diskutiert werden - Entwicklungen nicht in Nordamerika, Großbritannien oder anderen komplett anderen notfallmedizinischen Welten und Rettungssystemen, sondern Entwicklungen bei uns, im deutschsprachigen Raum.

Die Besonderheiten unserer weltweit beispielhaft hoch entwickelten Notfallmedizin finden sich bisweilen nicht in hochrangigen internationalen Fachzeitschriften, weil internationale Erkenntnisse nicht selten auf ein so hoch entwickeltes und notarztbesetztes Rettungssystem nur bedingt übertragbar sind. Auch deshalb werden Foren sehr gerne angenommen und unbedingt benötigt, in denen auch die hiesigen Spezifika vorgestellt werden, und auch damit kann man Impactpunkte generieren.

Was für Positionspapiere und Strukturdiskussionen zutrifft, das gilt auch für neue wissenschaftliche Erkenntnisse und Originalarbeiten. Diese sollten immer auch für unsere Notfallmedizin relevant sein, und die Daten sollten somit auch aus unserem System generiert worden sein.

Originalarbeiten aus dem deutschsprachigen Raum sind deshalb für uns unverzichtbar. Auch wenn die Arbeiten - wie im vorliegenden Heft - von retrospektivem Design sind, so beleuchten und analysieren sie auf einem sehr guten wissenschaftlichen Niveau wichtige Aspekte und Lösungen, die bei und für uns hochrelevant sind.

Für alle Autoren bietet eine Publikation in einer deutschsprachigen Zeitschrift immer auch ein individuelles Forum vor einem sehr nahen Leserkreis aus der gleichen Erfahrungswelt. Immer wieder nehmen solche Publikationen Einfluss auf die Entwicklung der Notfallmedizin und machen die Autoren in „ihrem“ beruflichen Um- und Entwicklungsfeld unmittelbar bekannt. Die hohe Reichweite - mit einer beeindruckenden Druckauflage von über 10.000 - und die ISI-Listung der Zeitschrift - mit dem Impact Factor von 0.529 - unterstützen zusätzlich maßgeblich den wichtigen und hohen Impact für den Autor.

In diesem Sinne freuen wir uns sehr, dass die große Zahl unser Leserinnen und Leser durch das Studium von Originalarbeiten in ihrer Fachlichkeit bereichert wird und die Autoren mit ihren Beiträgen hier auf sehr großes Interesse stoßen. Dass dies auch unmittelbar für die Autoren spürbar ist, zeigt die zunehmende Zahl an eingereichten hochkarätigen Originalarbeiten und der daraus resultierende zunehmend geringere Anteil von Annahmen zur Publikation durch unsere Gutachterinnen und Gutachter, der als weiteres Maß für die hohe Qualität angesehen werden kann.

In diesem Sinne wünschen wir Ihnen allen sehr herzlich eine interessante und bereichernde Lektüre der Arbeiten des vorliegenden Hefts.

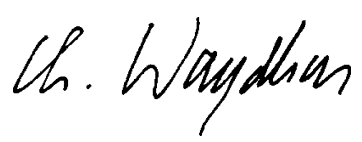

Christian Waydhas

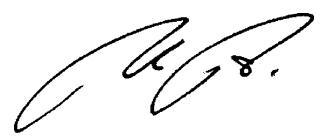

Bernd W. Böttiger

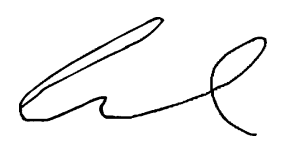

Christian Wrede

\section{Korrespondenzadresse}

\section{Prof. Dr. C. Waydhas}

Klinik und Poliklinik für Unfallchirurgie,

Universitätsklinikum Essen

Hufelandstraße 55, 45122 Essen, Deutschland

christian.waydhas@uni-essen.de

Interessenkonflikt. B.W. Böttiger ist European Resuscitation Council (ERC) Board Director Science and Research; Vorstandsvorsitzender des Deutschen Rates für Wiederbelebung/German Resuscitation Councils (GRC); Mitglied der Advanced Life Support (ALS) Task Force des International Liaison Committee on Resuscitation (ILCOR); Mitglied im Präsidium der Deutschen Interdisziplinären Vereinigung für Intensiv- und Notfallmedizin (DIVI), Associated Editor des European Journal of Anaesthesiology (EJA), Mitherausgeber der Zeitschrift Resuscitation. Für Vorträge hat er Honorare der folgenden Firmen erhalten: Medupdate, Forum für Medizinische Fortbildung (FoMF), Baxalta, Bayer Vital, Zoll, BARD. C. Waydhas und C. Wrede geben an, dass kein Interessenkonflikt besteht. 